\title{
Overexpression of adenylate cyclase-associated protein 1 is associated with metastasis of lung cancer
}

\author{
MIN TAN, XIAOLIAN SONG, GUOLIANG ZHANG, AIMEI PENG, \\ XUAN LI, MING LI, YANG LIU and CHANGHUI WANG
}

Department of Respiratory Medicine, Shanghai Tenth People's Hospital, Tongji University, Shanghai 200072, P.R. China

Received April 11, 2013; Accepted May 14, 2013

DOI: $10.3892 /$ or.2013.2607

\begin{abstract}
Lung cancer ranks first in both prevalence and mortality rates among all types of cancer. Metastasis is the main cause of treatment failure. Biomarkers are critical to early diagnosis and prediction and monitoring of progressive lesions. Several biomarkers have been identified for lung cancer but none have been routinely used clinically. The present study assessed the diagnostic and prognostic value of cyclase-associated protein 1 (CAP1) for lung cancer. CAP1 mRNA abundance and protein content were determined by real-time PCR and western blot analysis and/or immunostaining in biopsy specimens (24 neoplastic and 6 non-neoplastic) freshly collected at surgical lung resection, in 82 pathologically banked lung cancer specimens and in cultured non-invasive (95-C) and invasive (95-D) lung cancer cells. Multivariate regression analysis was performed to correlate immunoreactive CAP1 signal with cancer type and stage. In vitro cell migration was performed to determine the effect of RNA interference-mediated CAP1 gene silencing on invasiveness of 95-D cells. These analyses collectively demonstrated that: i) both CAP1 mRNA abundance and protein content were significantly higher in neoplastic compared to non-neoplastic specimens and in metastatic compared to non-metastatic specimens but not different between adenocarcinoma and squamous cell carcinoma; ii) immunoreactive CAP1 signal was significantly stronger in metastatic specimens and 95-D cells compared to non-metastatic specimens and 95-C cells; and iii) RNA interference-mediated CAP1 gene silencing adequately attenuated the invasive capacity of 95-D cells in vitro. These findings suggest that overexpression of CAP1 in lung cancer cells, particularly at the metastatic stage, may have signifi-
\end{abstract}

Correspondence to: Professor Changhui Wang, Department of Respiratory Medicine, Shanghai Tenth People's Hospital, Tongji University, 301 Middle Yanchang Road, Shanghai 200072, P.R. China

E-mail:wangch63@aliyun.com

Key words: lung cancer, metastasis, adenylate cyclase-associated protein, cyclase-associated protein 1 , small interfering RNA cant clinical implications as a diagnostic/prognostic factor for lung cancer.

\section{Introduction}

Lung cancer is a significant health problem not only in China, but worldwide. According to the information presented in the online database GLOBOCAN 2008 (http:/globocan.iarc. fr/factsheet.asp) of the International Agency for Research on Cancer, lung cancer was the most common and most aggressive cancer worldwide in 2008 , accounting for $16.5 \%$ of the total cancer cases and $22.5 \%$ of the total cancer-related deaths in 182 countries. In China, the incidence and mortality rates of lung cancer also rank first among all types of cancer, which were predicted to be 33.5/100,000 and 28.7/100,000, respectively, in 2008 based on the data from 36 cancer registries and the Third National Death Survey (2004-2005) (1).

Approximately $85 \%$ of lung cancer cases are non-small cell lung cancer (NSCLC), which includes adenocarcinoma (AD), squamous cell carcinoma (SCC), large cell carcinoma and bronchioloalveolar carcinoma (2). Despite extensive research and clinical efforts, prognosis of NSCLC remains poor. Nevertheless, biomarkers have been recognized to play a critical role in early diagnosis, therapy guidance and prognosis monitoring of NSCLC (3). Over the last two decades, several biomarkers have been identified for NSCLC, but none have been routinely used in clinical settings (4). It is, therefore, urgent to explore more reliable and feasible novel biomarkers for lung cancer.

Adenylate cyclase-associated protein 1 (CAP1) is an actin monomer-binding protein coded by the CAP1 gene (5), which was originally cloned from budding yeast and is located in the downstream of the ras gene (6). Human homology of CAP1 was identified in the early 1990s (7). Both mammal and yeast CAPs interact with actin (8) and play a role in actin turnover (9). Given the critical role for actin filament reorganization in cell migration and the regulatory role for CAP1 in actin filament reorganization $(10,11)$, it is logical to hypothesize that CAP1 may be associated with tumor metastasis. Currently, however, studies on the correlation between CAP1 expression and tumor metastasis are scarce (12). The objective of the present study was to evaluate the potential value of CAP1 in early diagnosis and prognostic prediction of progressive lung cancer. 


\section{Materials and methods}

Ethical considerations. The study was reviewed and approved by the Institutional Ethics Committee of Tongji University School of Medicine in Shanghai and was conducted in compliance with the Helsinki Declaration. Written informed consent was obtained from all subjects.

Subjects and biopsy specimen collection. Twenty-four lung cancer patients and 6 control subjects with non-neoplastic lung condition(s) who underwent surgical lung resection of neoplastic and non-neoplastic lesions, respectively, at the Shanghai 10th People's Hospital, China, between 2007 and 2009 were recruited. Cancer types and stages were determined based on results from laboratory tests, X-rays, CT scans, bone scans and MRI scans. Biopsy specimens were collected at surgery, snap-frozen in liquid nitrogen and stored at $-80^{\circ} \mathrm{C}$ until analyses.

Tissue bank specimen acquisition. Tissue blocks of 82 formalin-fixed and paraffin-embedded lung cancer specimens were acquired from the Tissue Bank in the Pathology Department of Tongji University School of Medicine. The documented general demographic data and cancer stages assessed based on the tumor-node-metastasis (TNM) classification system were obtained.

Lung cancer cell line. For in vitro analyses, a low invasive lung cancer cell line 95-C and an invasive lung cancer cell line 95-D were purchased from the American Type Culture Collection (ATCC, Manassas, VA, USA). For maintenance, cells were cultured in RPMI-1640 supplemented with $10 \%$ fetal bovine serum (FBS) at $37^{\circ} \mathrm{C}$ in a humidified atmosphere of $5 \% \mathrm{CO}_{2}$ and $95 \%$ air.

Real-time PCR. Total RNA was isolated from neoplastic and non-neoplastic lung biopsy specimens using an RNA extraction kit from Isogen (Nippon Gene Co., Ltd., Toyama, Japan). RNA samples were treated with DNase I (Promega Corp., Madison, WI, USA) to remove genomic DNA. First-strand cDNAs were synthesized using a commercial First-Strand cDNA Synthesis kit as instructed by the manufacturer. PCR amplifications of the test gene CAP1 and the reference gene glyceraldehyde-3-phosphate dehydrogenase (GAPDH) were performed using, respectively, a pair of primers (forward, 5'-ACT CGC TGC TTG CTG GTC-3' and reverse: 5'-ATG GGT GCC AAC AAA TCG-3') designed based on the human CAP1 mRNA sequence (GenBank accession number BT007152) and a pair of primers (forward, 5'-GAA GGT GAA GGT CGG AGTC-3' and reverse, 5'-CCC GAA TCA CAT TCT CCA AGA A-3) designed based on the human GAPDH cDNA sequence (GenBank access number X01677). All reactions were carried out with the SYBR-Green PCR Core Reagents kit (Perkin-Elmer Applied Biosystems, Foster City, CA, USA). The PCR amplification parameters were: $50^{\circ} \mathrm{C}$ for $2 \mathrm{~min}$ (one cycle), $95^{\circ} \mathrm{C}$ for $10 \mathrm{~min}$ (one cycle), $95^{\circ} \mathrm{C}$ for $15 \mathrm{sec}$ and $60^{\circ} \mathrm{C}$ for $1 \mathrm{~min}$ (40 cycles). The emission intensity of the SYBRGreen fluorescence was measured in a real-time fashion with the ABI Prism 7700 Sequence Detector from Perkin-Elmer Applied Biosystems. Relative quantitation of CAP1 mRNA abundance was performed using the DataAssist software (Life Technologies, Grand Island, NY, USA).

Western blot analysis. Whole cell and tissues lysates were prepared from cultured 95-C and 95-D cells and biopsy lung specimens, respectively. Following total protein quantitation, the lysates were loaded onto $10 \%$ polyacrylamide-SDS gels, separated by electrophoresis and blotted onto N-C membrane blots using a semi-dry transfer system. Blots were incubated with a mouse anti-human CAP1 antibody and a mouse antihuman actin antibody (Sigma, St. Louis, MO, USA) at $4^{\circ} \mathrm{C}$ overnight. After washing, blots were incubated with horseradish peroxidase-conjugated secondary antibodies at room temperature for $45 \mathrm{~min}$. The immunoreactive signals for both CAP1 and actin were visualized using the ECL system from GE Healthcare UK Ltd. (Buckinghamshire, UK) and subjected to densitometric analyses with the ImageJ software (National Institutes of Health, Bethesda, MD, USA). Relative levels of CAP1 (i.e., after adjustment against actin) were determined based on the densitometric data.

Immunohistochemical and immunocytochemical staining. Lung cancer specimen blocks obtained from the tissue bank were sectioned at $5 \mu \mathrm{M}$ and mounted onto glass slides. Low and high invasive lung cancer 95-C and 95-D cells were grown on culture slides (BD Biosciences, San Diego, CA, USA) and fixed in $3.7 \%$ formaldehyde in PBS. Both tissue sections and cultured cells were incubated with a primary CAP1 antibody at $4^{\circ} \mathrm{C}$ overnight. After rinsing three times in Tris-buffered saline $(0.01 \mathrm{M}$ Tris- $\mathrm{HCl} \mathrm{pH} 7.5,150 \mathrm{mM}$ $\mathrm{NaCl}$ ), tissue sections and cultured cells were incubated with FITC-labeled secondary antibody (Invitrogen, Carlsbad, CA, USA) at room temperature for $30 \mathrm{~min}$. After washing, the slides were mounted with a DAPI-containing mounting solution to identify cell nuclei. Fluorescent signals were visualized under an Axiovert 200 microscope and images were captured using an AxioCam CCD camera and the AxioVision software (Leica Microsystems, Wetzlar, Germany). The human peritumoral vascular endothelial cells were used as a positive control.

Staining results were assessed by two pathologists, blind to the specimen identity, independently. The immunoreactive intensity in the test tissue specimens and cultured tumor (95-C and 95-D) cells was graded by the difference against the intensity in endothelial cells in the positive control group: 0 , negative; 1 (weak), weaker than epithelial cells; 2 (moderate), the same as epithelial cells; 3 (strong), stronger than epithelial cells. A staining score of 2 or 3 was considered CAP1-positive.

RNA interference. Invasive 95-D cells were seeded onto collagen I-coated 6 -well plates at $5 \times 10^{4}$ cells/well. Following overnight culture, cells were transfected with CAP1 specific siRNA (siCAP1) or non-silencing control siRNA in serumfree RPMI-1640 using Oligofectamine (Invitrogen). The target sequence was AAACCGAGTCCTCAAAGAGTA. Both specific and control siRNA molecules were synthesized by GenePharma Co. (Shanghai, China). Four hours after transfection, an equal volume of medium supplemented with $20 \%$ fetal bovine serum (FBS) was added. Transfectants were cultured until further analysis. 
Table I. General demographics and CAP1 immunoreactive signals in the 82 patients whose lung tumor tissue specimens were banked in the institutional tissue bank.

\begin{tabular}{|c|c|c|c|c|}
\hline Variables & Total N (\%) & CAP1-negative & CAP1-positive & P-value \\
\hline Age (years) & & & & 0.204 \\
\hline$\leq 65$ & $43(52.4)$ & 15 & 28 & \\
\hline$>65$ & $39(47.6)$ & 19 & 20 & \\
\hline Gender & & & & 0.701 \\
\hline Female & $27(32.9)$ & 12 & 15 & \\
\hline Male & $55(67.1)$ & 22 & 33 & \\
\hline Histologic type & & & & 0.526 \\
\hline $\mathrm{AD}$ & $40(48.8)$ & 18 & 22 & \\
\hline $\mathrm{SCC}$ & $31(37.8)$ & 16 (non-AD) & 26 (non-AD) & \\
\hline Others & $11(13.4)$ & & & \\
\hline Tumor size $(\mathrm{T})(\mathrm{cm})$ & & & & 0.231 \\
\hline$\leq 3$ & $37(45.1)$ & 18 & 19 & \\
\hline$>3$ & $45(54.9)$ & 16 & 29 & \\
\hline Node metastasis $(\mathrm{N})$ & & & & 0.01 \\
\hline $0-1$ & $42(51.2)$ & 22 & 20 & \\
\hline $2-3$ & $40(48.8)$ & 12 & 28 & \\
\hline Distant metastasis (M) & & & & 0.126 \\
\hline 0 & $63(76.8)$ & 29 & 34 & \\
\hline 1 & $9(23.2)$ & 5 & 14 & \\
\hline TNM & & & & 0.04 \\
\hline I & & 17 & 8 & \\
\hline II-IV & & 17 & 40 & \\
\hline
\end{tabular}

CAP1, cyclase-associated protein 1; AD, adenocarcinoma; SCC, squamous cell carcinoma; TNM, tumor-node-metastasis.

Migration assay. Invasive 95-D cells transfected with siCAP or control siRNA were seeded onto the upper chambers of 6-well transwell plates (BD Biosciences, San Jose, CA, USA) in triplicates in RPMI-1640 supplemented with $0.5 \%$ FBS. Culture medium containing $10 \%$ FBS was added to the bottom chamber. After a 24-h culture, cells on the upper surface of the chamber membrane were removed and those on the lower surface of the membrane were stained by hematoxylin and eosin (H\&E). The number of migrated cells was counted under a light microscope $(x 400)$.

Statistical analysis. Categorical data expressed as percentage were analyzed by the $\chi^{2}$ test. Correlations between CAP1 expression and various clinicopathological variables was analyzed by multivariate logistic regression analysis. The statistical software SPSS version 17.0 (SPSS Inc., Chicago, IL, USA) was used. Differences were considered significant when $\mathrm{P}<0.05$.

\section{Results}

Demographic characteristics. In the present study, 24 lung cancer patients and 6 non-neoplastic control subjects were recruited and 82 tissue bank specimens of lung cancer were included. Of the 24 lung cancer patients, 4 had advanced (metastasis), 10 were of the SCC and 14 of the AD type. The base-line demographic data on the 82 tissue bank cases are presented in Table I.

CAP1 mRNA abundance. To assess the association of CAP1 expression with lung cancer type and stage, we first performed real-time PCR to analyze relative CAP1 mRNA abundance in non-neoplastic and lung cancer biopsy specimens. The results are shown in Fig. 1. The relative CAP1 mRNA abundance, expressed as the CAP1/GAPDH ratio, was significantly higher in neoplastic tissues than in control specimens $(\mathrm{P}=0.028)$, and in metastatic than in non-metastatic lung cancer specimens $(\mathrm{P}=0.016)$. When a comparison was made between histological types, SCC specimens had slightly higher CAP1 mRNA abundance than AD specimens, but the difference failed to reach a statistically significant level $(\mathrm{P}=0.227)$.

CAP1 protein levels. In addition, mRNA abundance, CAP1 protein levels in biopsy specimens and cultured cells were determined by western blot analysis. Shown in Fig. 2 is an image of a representative blot. When expressed as a densitometric ratio of CAP1 band to $\beta$-actin band, the relative CAP1 protein level was significantly elevated in lung cancer patients $(0.7527 \pm 0.2767)$ as compared to non-neoplastic control subjects $(0.3476 \pm 0.1713, \mathrm{P}=0.002)$; it was also significantly elevated in metastatic lung tumors $(0.8941 \pm 0.1442)$ as compared to non-metastatic lung tumors $(0.4701 \pm 0.2647$, 


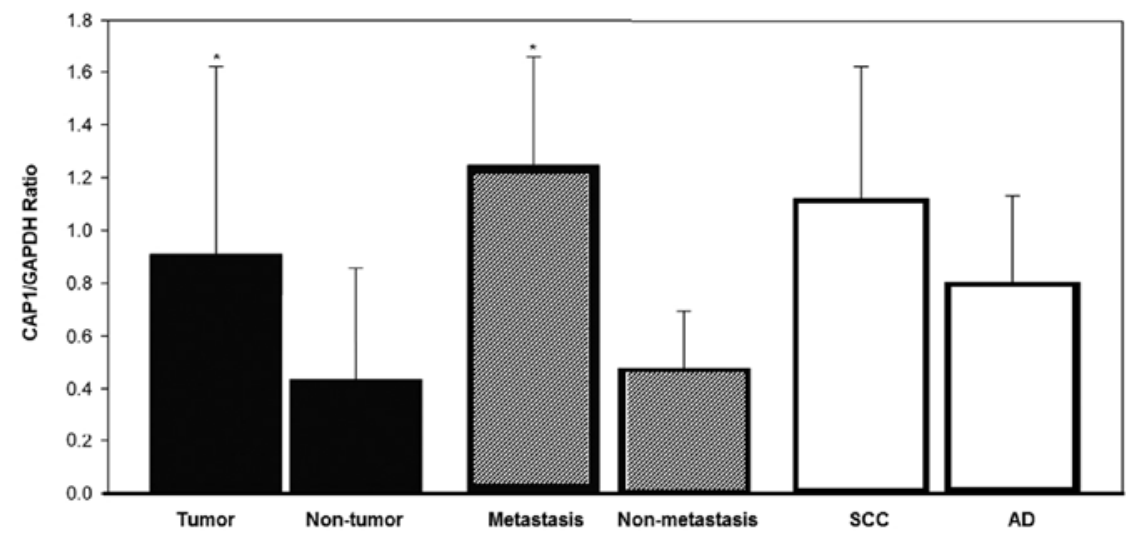

Figure 1. Relative cyclase-associated protein 1 (CAP1) mRNA levels (i.e., CAP1/GAPDH mRNA ratio) in non-neoplastic lung tissue and lung tumors of different stage and histological type. ${ }^{*} \mathrm{P}=0.05$, comparison made within the same type of bar.

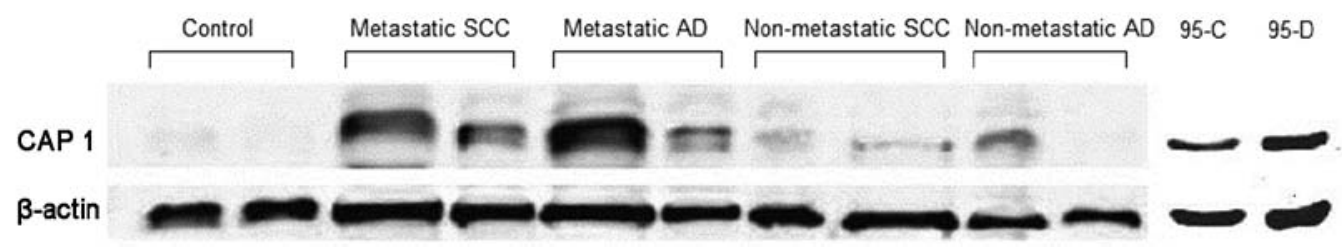

Figure 2. A representative western blot analysis showing cyclase-associated protein 1 (CAP1) and $\beta$-actin bands in indicated biopsy specimens (two representative samples from each of the patient groups) and cultured lung cancer cells.
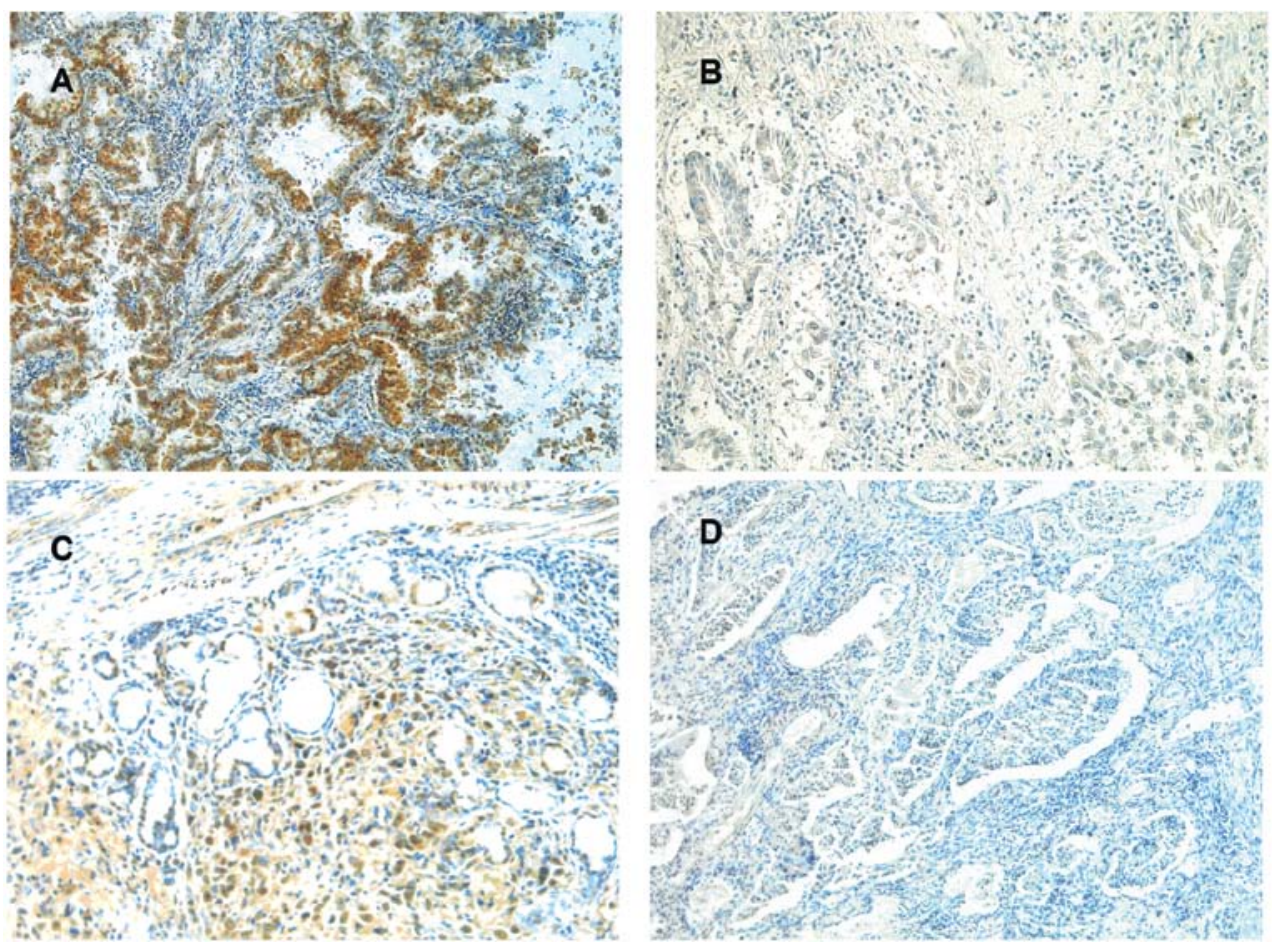

Figure 3. Representative sections of pathological lung cancer specimens after immunohistochemical staining. (A) Adenocarcinoma (AD) showing a high percentage of cyclase-associated protein 1 (CAP1)-positive cells; (B) AD showing a low percentage of CAP1-positive cells; (C) squamous cell carcinoma (SCC) showing a high percentage of CAP1-positive cells; (D) SCC showing a low percentage of CAP1-positive cells.

$\mathrm{P}=0.002)$; it was slightly higher in SCC than in AD specimens ( $0.7440 \pm 0.2911$ vs. $0.7601 \pm 0.2757, \mathrm{P}=0.891)$, but the difference was not significant $(\mathrm{P}>0.05)$. In cultured cell lines, the CAP1 protein level was significantly higher in invasive 95-D cells than that in non-invasive 95-C cells $(\mathrm{P}<0.05)$.
Correlation between immunoreactive CAP1 signals and cancer metastasis. Immunoreactive CAP1 was detected in the cytoplasm of cancer cells in all 82 tissue bank samples (Fig. 3) and cultured 95-C and 95-D cells and also in the cytoplasm of stromal cells (Fig. 4). Using the signal in the positive control 

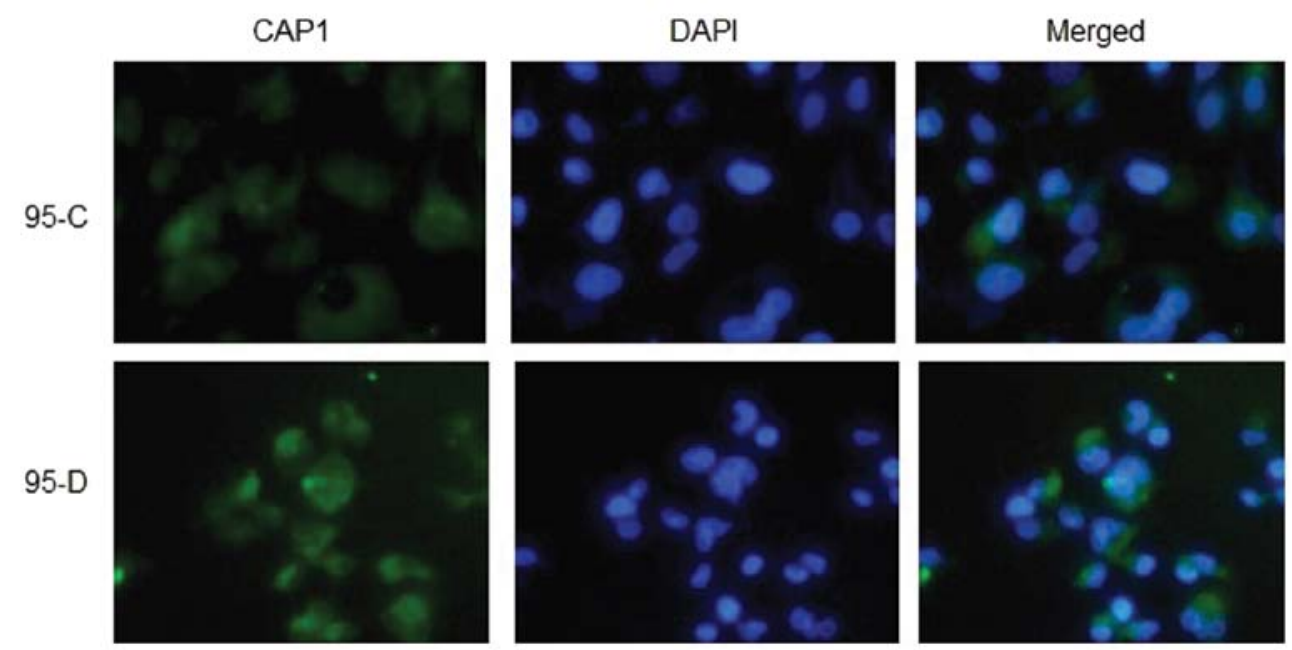

Figure 4. A representative image of non-invasive and invasive 95-C and 95-D lung cancer cells following immunostaining with anti-cyclase-associated protein 1 (CAP1) antibody and nuclear staining with DAPI.

A

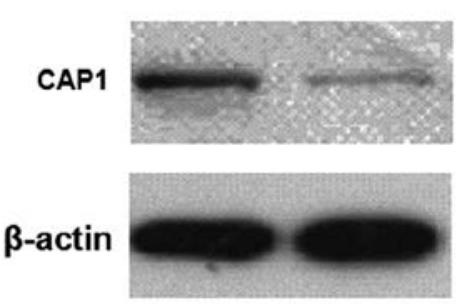

B

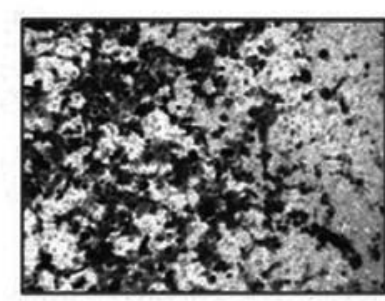

CAP1 SIRNA

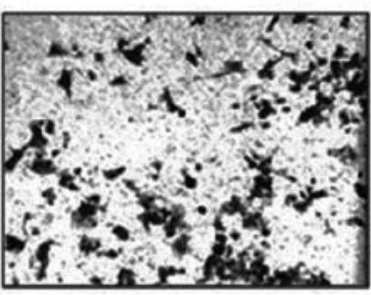

Figure 5. A representative image of invasive 95-D lung cancer cells transfected with the control siRNA and the cyclase-associated protein 1 (CAP1)-specific siRNA respectively after migrating over the transwell membrane in the in vitro cell migration assay. Migrated cells were stained.

endothelial cells as a reference, the 82 bank samples were classified into a CAP1-negative group, where the tumor staining score was 0 or 1 , and a CAP1-positive group, where the tumor staining score was 2 or 3 . As shown in Table I, a CAP1-positive status was significantly associated with lymph node metastasis $(\mathrm{P}=0.04)$ and $\mathrm{TNM}$ stage $(\mathrm{P}=0.01)$ as demonstrated by the $\chi^{2}$ test.

To assess the association of CAP1 expression simultaneously with various demographic variables and tumor properties in lung cancer cases, multivariate logistic regression analysis was performed. These results indicated that the expression level of CAP1 was associated with TNM stage of lung cancer $(\mathrm{P}=0.01)$, particularly the lymph node invasion $(\mathrm{P}=0.04)$ (Table I).

Reduced migration of invasive lung cancer cells following CAP1 knockdown. To validate the positive correlation between CAP1 and metastasis of lung tumors, we attempted to silence the CAP1 gene in invasive lung cancer 95-D cells with a small interfering RNA (siRNA) molecule specific for human CAP1 and to determine the migration capacity of the CAP1-siRNA carrying cells in vitro. As shown in Fig. 5, the CAP1 gene was adequately silenced by sequence-specific CAP1-siRNA and correspondingly the number of cells that migrated across the transwell membrane was significantly reduced in the CAP1 gene-silenced 95-D cells.

\section{Discussion}

As a first step toward evaluating the association of CAP1 with histological type and clinical stage of lung cancer, we first assessed the CAP1 gene expression at the mRNA level in 24 lung cancer patients and 6 non-cancer control subjects by real-time PCR. The results clearly demonstrated that the CAP1 gene transcription was significantly elevated in lung cancer patients and the elevation was more pronounced in metastasis (Fig. 1).

Although it is generally assumed that mRNA abundance is predictive of the corresponding protein level, this assumption may not be valid for all human genes. In a study aimed at assessing the abundance of 165 protein spots representing 98 individual genes using 76 lung adenocarcinomas and 9 non-neoplastic lung tissue specimens, marked variation in the mRNA/protein correlation coefficient was found among proteins and between stage I and stage III lung adenocarcinomas (13). To further analyze whether the observed elevation in CAP1 mRNA abundance was correlated with increased CAP1 protein synthesis, we next performed western blot analysis on the CAP1 protein content in biopsy specimens and cultured lung cancer cells. In accordance with the pattern of changes in CAP1 mRNA abundance in various specimens, the CAP1 protein content was significantly higher in cancer than in non-cancer subjects and higher in metastatic than in 
non-metastatic cancer patients with no difference between SCC and AD (Fig. 2). Since the sample size used in the present study was small and there are no reports in the literature on the CAP1 mRNA/protein correlation coefficient, more thorough studies with larger sample sizes are warranted to validate the concurrent elevation in CAP1 mRNA and protein levels in lung cancer.

To further evaluate the correlation between CAP1 expression and histological type and clinical stage of lung cancer, we performed immunostaining on 82 tissue bank specimens and cultured 95-C and 95-D cells. Despite the lack of reports on intracellular localization of CAP1 protein in lung cancer cells, non-nuclear localization of CAP1 has been observed at the site of rapid actin turnover in non-muscle cells (9). In the present study, stronger immunoreactive CAP1 signals were detected in the perinuclear cytoplasm of lung cancer cells in the biopsy specimens (Fig. 3) and cultured lung cancer cells (Fig. 4). This intracellular localization suggests that CAP1 may be associated with lamellipodium formation and cell migration. Following classification of the 82 tissue bank specimens as CAP1-negative and CAP1-positive, two groups based on their immunoreactive signal densities relative to the density in endothelial cells in the positive control group and a subsequent multivariate regression analysis, the CAP1 immunoreactive signal was positively correlated with metastasis, particularly metastasis to lymph nodes (Table I). Given that lymph node metastasis of lung cancer is associated with poor clinical outcomes $(14,15)$, this finding suggests that immunostaining of the CAP1 protein in lung cancer tissue may provide additional information predictive of patient prognosis.

siRNA-based RNA interference (RNAi) is a post-transcriptional process triggered by the introduction of double-stranded RNA (dsRNA) which leads to gene silencing in a sequencespecific manner (16). As one of the most notable discoveries of the past decade in functional genomics, RNAi has become an important tool for analyzing gene functions in eukaryotes. Using this advanced technology, we successfully silenced the CPA1 gene in invasive lung cancer 95-D cells (Fig. 4), which resulted in a significant reduction in the capacity of 95-D cells to migrate (Fig. 5). This observation further supports a functional role for CAP1 in the process of lung cancer cell metastasis.

At present, the molecular mechanisms underlying the effect of CAP1 on lung cancer cell migration and metastasis are largely unknown. Cofilin is a family of actin-binding proteins which disassembles actin filaments. Cofilin activity is present in the malignant, invasive cancer cells (17) and may function as a factor to regulate cancer cell migration/invasion phenotypes. We previously demonstrated that CAP1 is a downstream target of cofilin (18), suggesting that the CAP1 overexpression-associated lung cancer cell migration and metastasis may involve a cofilin-mediated signaling pathway. Nevertheless, this requires further evaluation.

\section{Acknowledgements}

The present study was supported by a research grant from the Shanghai Science Committee (09ZR1424500).

\section{References}

1. Chang S, Dai M, Ren JS, Chen YH and Guo LW: Estimates and prediction on incidence, mortality and prevalence of lung cancer in China in 2008. Zhonghua Liu Xing Bing Xue Za Zhi 33: 391-394, 2012 (In Chinese)

2. Ramalingam SS, Owonikoko TK and Khuri FR: Lung cancer: new biological insights and recent therapeutic advances. CA Cancer J Clin 61: 91-112, 2011.

3. Cho JY and Sung HJ: Proteomic approaches in lung cancer biomarker development. Expert Rev Proteomics 6: 27-42, 2009.

4. Valle RP, Chavany C, Zhukov TA and Jendoubi M: New approaches for biomarker discovery in lung cancer. Expert Rev Mol Diagn 3: 55-67, 2003.

5. Fedor-Chaiken M, Deschenes RJ and Broach JR: SRV2, a gene required for RAS activation of adenylate cyclase in yeast. Cell 61: 329-340, 1990.

6. Field J, Vojtek A, Ballester R, et al: Cloning and characterization of $C A P$, the $S$. cerevisiae gene encoding the $70 \mathrm{kd}$ adenylyl cyclase-associated protein. Cell 61: 319-327, 1990.

7. Matviw H, Yu G and Young D: Identification of a human cDNA encoding a protein that is structurally and functionally related to the yeast adenylyl cyclase-associated CAP proteins. Mol Cell Biol 12: 5033-5040, 1992.

8. Freeman NL, Chen Z, Horenstein J, Weber A and Field J: An actin monomer binding activity localizes to the carboxyl-terminal half of the Saccharomyces cerevisiae cyclase-associated protein. J Biol Chem 270: 5680-5685, 1995.

9. Moriyama K and Yahara I: Human CAP1 is a key factor in the recycling of cofilin and actin for rapid actin turnover. J Cell Sci 115: 1591-1601, 2002.

10. Hubberstey AV and Mottillo EP: Cyclase-associated proteins: CAPacity for linking signal transduction and actin polymerization. FASEB J 16: 487-499, 2002.

11. Loisel TP, Boujemaa R, Pantaloni D and Carlier MF: Reconstitution of actin-based motility of Listeria and Shigella using pure proteins. Nature 401: 613-616, 1999.

12. Yamazaki K, Takamura M, Masugi Y, et al: Adenylate cyclaseassociated protein 1 overexpressed in pancreatic cancers is involved in cancer cell motility. Lab Invest 89: 425-432, 2009.

13. Chen G, Gharib TG, Huang CC, et al: Discordant protein and mRNA expression in lung adenocarcinomas. Mol Cell Proteomics 1: 304-313, 2002.

14. Renyi-Vamos F, Tovari J, Fillinger J, et al: Lymphangiogenesis correlates with lymph node metastasis, prognosis, and angiogenic phenotype in human non-small cell lung cancer. Clin Cancer Res 11: 7344-7353, 2005

15. Sun JG, Wang Y, Chen ZT, et al: Detection of lymphangiogenesis in non-small cell lung cancer and its prognostic value. J Exp Clin Cancer Res 28: 21, 2009.

16. Macrae IJ, Zhou K, Li F, et al: Structural basis for doublestranded RNA processing by Dicer. Science 311: 195-198, 2006.

17. Van Troys M, Huyck L, Leyman S, Dhaese S, Vandekerkhove J and Ampe C: Ins and outs of ADF/cofilin activity and regulation. Eur J Cell Biol 87: 649-667, 2008.

18. Wang W, Eddy R and Condeelis J: The cofilin pathway in breast cancer invasion and metastasis. Nat Rev Cancer 7: 429-440, 2007. 